\title{
Male accessory breast cancer successfully treated with endocrine therapy: A case report
}

\author{
LINTAO BI, JUN LI, ZHANGZHEN SHI, ZHENXING ZHU and ZHENXIA LU \\ Department of Hematology and Oncology, China-Japan Union Hospital Affiliated to Jilin University, \\ Changchun, Jilin 130033, P.R. China
}

Received September 24, 2014; Accepted May 20, 2015

DOI: $10.3892 / \mathrm{ol} .2015 .3602$

\begin{abstract}
Male accessory breast cancer is an extremely rare tumor. Several risk factors have been identified, including genetic and hormonal abnormalities. Accessory breast carcinoma usually occurs under the axilla or in the inguinal region. Clinical diagnosis is frequently delayed due to the general lack of awareness among physicians and patients. In the present study, the case of a 63-year-old male patient who was diagnosed with accessory breast cancer at a local advanced stage was reported. However, the patient was successfully treated with endocrine therapy.
\end{abstract}

\section{Introduction}

Breast cancer in males is extremely rare and accounts for $\sim 1 \%$ of all malignant breast neoplasm cases $(1,2)$. Accessory breasts are observed in $2-6 \%$ of the general population (3), and these tissues may present in various positions along the milk line, but most frequently in the axillary region (4). Accessory breast carcinoma is a rare form of breast cancer usually occurring in the axilla or inguinal region, where there are abundant lymph nodes and capillaries, and the incidence rate is $0.2-0.6 \%$ (5). There are few cases of accessory breast carcinoma in males reported in the literature (6-8). The principal malignancy identified in accessory breast tissue, as with normal breasts, is invasive ductal carcinoma (79\%). In the majority of previously documented cases, treatment regimens for accessory breast carcinoma follow the guidelines for breast cancer (9-11). Early diagnosis of this carcinoma is difficult due to its rarity and a general lack of awareness among physicians and patients $(9,10,12)$. Thus, metastasis occurs at an early stage and the prognosis of patients is often poor, however, due to limited follow-up data and small sample sizes of previous studies, an

Correspondence to: Dr Zhenxia Lu, Department of Hematology and Oncology, China-Japan Union Hospital Affiliated to Jilin University, 5th Building, 126 XianTai Street, Changchun, Jilin 130033, P.R. China

E-mail: bilintao@sohu.com

Key words: male accessory breast cancer, endocrine therapy accurate prognosis for accessory breast carcinoma is difficult to estimate (12).

The present study reported the case of a 56-year-old male accessory breast cancer patient who underwent a series of four surgical excisions of a primary ectopic breast carcinoma and developed local lymph node and opposite supraclavicular lymph node metastasis. Subsequently, the patient developed pulmonary and bone metastasis. The patient was successfully treated with an endocrine therapy regimen (anastrozole and goserelin). Furthermore, the present study evaluated the best approach for the treatment of accessory breast neoplasm in male patients.

\section{Case report}

A 56-year-old Chinese male was referred to a local hospital in July 2005, complaining of a mung bean-sized mass under the right axilla. The patient underwent mass resection; however, the resected tissue was not pathologically examined as it was considered to be lipoma by the doctor, and thus no official diagnosis was recorded. On April 23rd, 2006, a second mung bean-sized mass was detected at the same site, which was again excised with no pathological examination. In October 2007, the patient presented at the China-Japan Union Hospital Affiliated to Jilin University (Changchun, China) with a further, yolk-sized painful mass at the same site. Tumor resection was performed, and the macroscopic appearance of the lesion indicated a poorly-differentiated adenocarcinoma in dermis and subcutaneous tissues. Immunohistochemical analysis [with (-), negative; $(+)$, positive; and $(+++)$, strongly positive] demonstrated that the mass was cytokeratin (CK)20 (-), estrogen receptor (ER) (+++), C-erbB-2 (-), thyroid transcription factor 1 (TTF-1) (-) and gross cystic disease fluid protein 15 (GCDFP-15) (focal+). According to the clinical performance and pathological findings, the mass was considered to be an adenocarcinoma from the accessory breast tissue. Next, the patient received right breast modified radical mastectomy and left breast simple excision. Postoperative pathology results indicated reactive hyperplasia of giant cell and fibrillar connective tissue, observed in the right breast. A total of 3 out of the 17 dissected axillary lymph nodes were positive for metastatic carcinoma. There was no cancer in the left breast tissue. The patient was diagnosed with right accessory breast carcinoma (PT1N1M0) (13), right axilla metastatic carcinoma and hypertrophy of the left breast. Subsequently, the patient received six cycles of adjuvant chemotherapy 


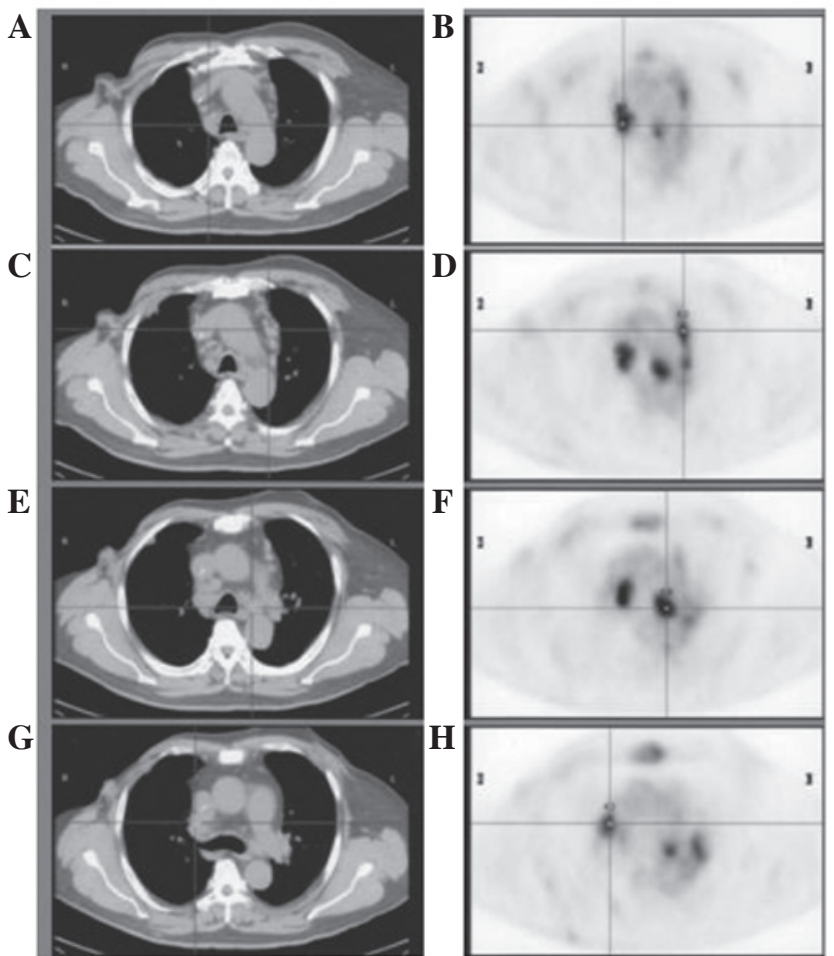

Figure 1. Positron emission tomography (right)-computed tomography (left) scans (May 24th, 2011) revealed multiple high metabolic enlarged lymph nodes in mediastina and increased ${ }^{18} \mathrm{~F}$-fluorodeoxyglucose uptake in the (A and B) station 4R, (C and D) station 6, (E and F) station $4 \mathrm{~L}$ and $(\mathrm{G}$ and $\mathrm{H})$ station $10 \mathrm{R}$ lymph nodes. Lymph node mapping was determined according to the IASLC lymph node map (14).
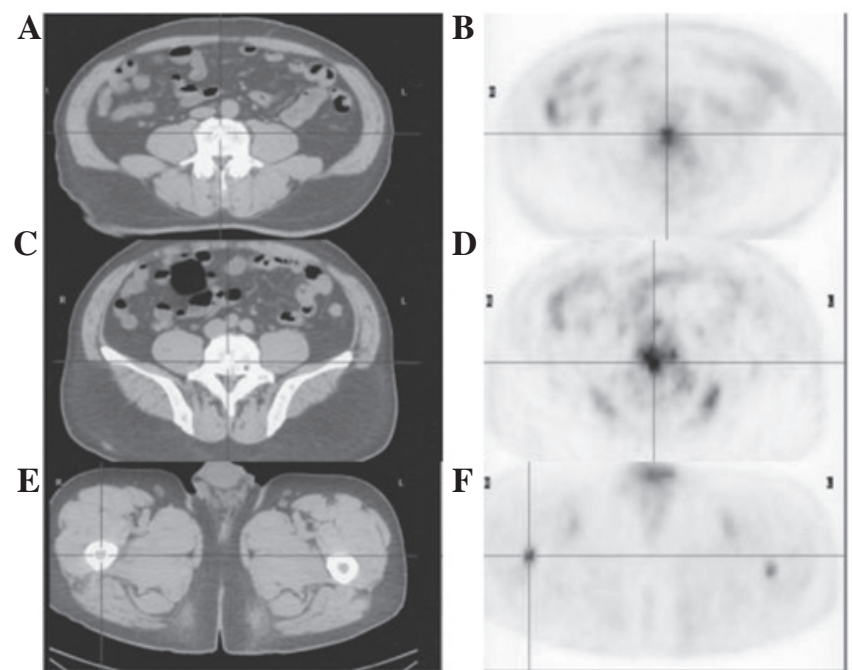

Figure 2. PET (right)-CT (left) scan (May 24th, 2011) revealed osteoblastic metastasis with increased ${ }^{18} \mathrm{~F}-\mathrm{FDG}$ uptake in the (A and B) lumbar and (C and D) sacral vertebrae. (E) No evident changes were identified on axial CT, however, (F) ${ }^{18}$ F-FDG uptake was observed on axial PET. CT, computed tomography; ${ }^{18} \mathrm{~F}$-FDG, ${ }^{18} \mathrm{~F}$-fluorodeoxyglucose; PET, positron emission tomography.

[docetaxel, $140 \mathrm{mg}$ intravenously (i.v.) and cyclophosphamide (CTX), 1 g i.v.; on day 1 then once every 3 weeks] along with radiation therapy.

On April 20th, 2011, the patient incidentally detected a further mung bean-sized mass adjacent to the his left subclavian
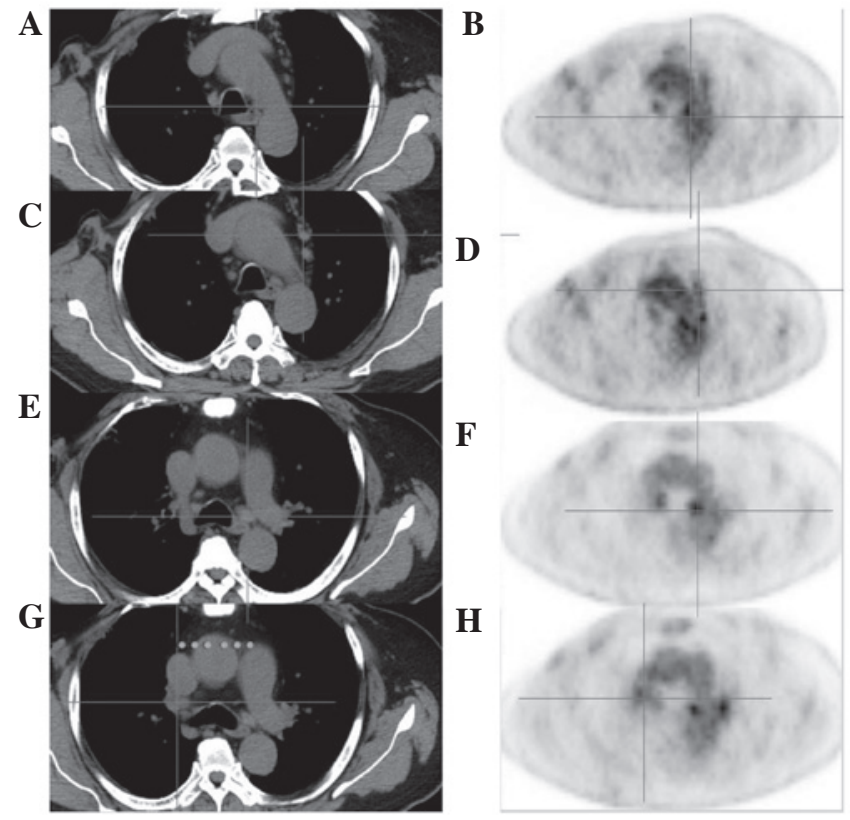

Figure 3. Positron emission tomography (right)-computed tomography (left) scans (May 24th, 2012) demonstrated that the enlarged mediastinal lymph nodes decreased in size, while ${ }^{18} \mathrm{~F}$-fluorodeoxyglucose uptake was also reduced in the (A and $\mathrm{B})$ station $4 \mathrm{R},(\mathrm{C}$ and $\mathrm{D})$ station 6 , (E and F) station $4 \mathrm{~L}$ and $(\mathrm{G}$ and $\mathrm{H})$ station 10R lymph nodes. Lymph node mapping was determined according to the IASLC lymph node map (14).
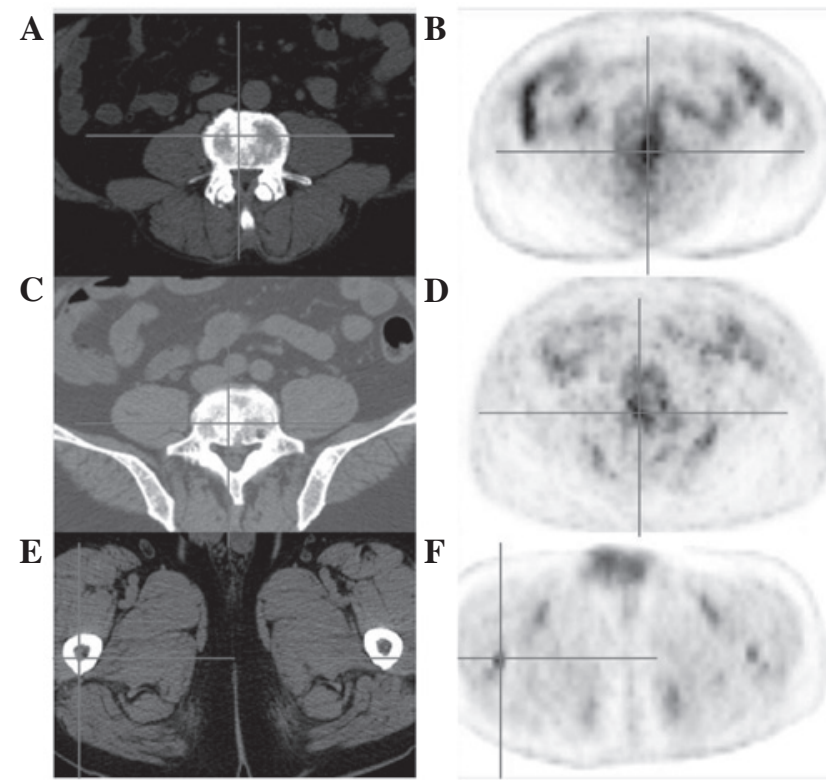

Figure 4. PET (right)-CT (left) scans (May 24th, 2012) revealed that the metabolism of osteoblastic metastases was reduced compared with that prior to endocrine therapy. No evident changes were observed on axial CT scans, however the corresponding PET scans revealed decreased ${ }^{18} \mathrm{~F}$-fluorodeoxyglucose uptake in the (A and B) lumbar and (C and D) sacral vertebrae and (E and $\mathrm{F}$ ) right femoral shaft. $\mathrm{CT}$, computed tomography; PET, positron emission tomography.

and, at the same time, experienced lower-limb bone pain. This mass was then resected and the pathological results indicated a poorly-differentiated metastatic carcinoma. Immunohistochemical analysis revealed that the mass was CK (focal+), vimentin (+), CK7 (+), CD117 (+), E-calpain (+), ER (3+), 
Table I. Laboratory test results.

Current case

\begin{tabular}{lccr} 
Markers & Normal range & Prior to therapy & 1 year after therapy \\
\hline Carcinoembryonic antigen, ng/ml & $0.50-9.60$ & 28.18 & 11 \\
Cancer antigen $15-3, \mathrm{U} / \mathrm{ml}$ & $0.10-31.30$ & 31.12 & 16 \\
Serum alkaline phosphatase, IU/1 & $30.0-120.0$ & 188.00 & 145
\end{tabular}
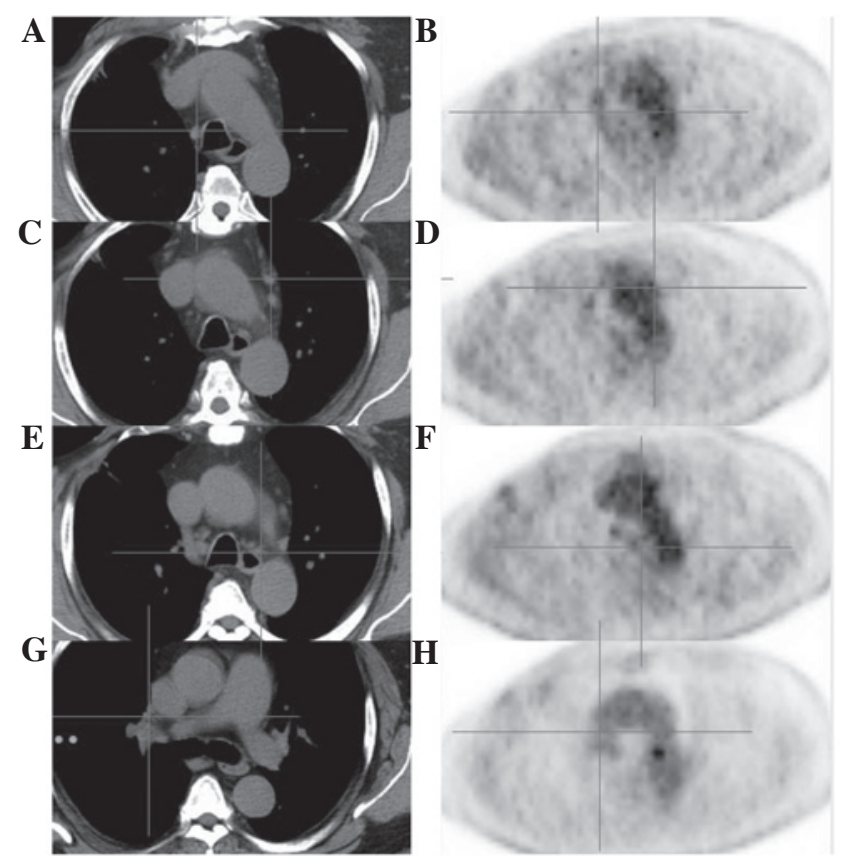

Figure 5. Positron emission tomography (right)-computed tomography (left) scans (March 10th, 2014) demonstrated that the enlarged lymph nodes decreased further in size, and the metabolic level was also reduced to normal in the (A and B) station $4 \mathrm{R},(\mathrm{C}$ and $\mathrm{D})$ station 6, (E and F) station $4 \mathrm{~L}$ and $(\mathrm{G}$ and $\mathrm{H})$ station 10R lymph nodes. Lymph node mapping was determined according to the IASLC lymph node map (14).

progesterone receptor (PR) (+++) and CD56 (+); however, the tissue was negative for TTF-1, Syn, HMB45, chromogranin A and human epidermal growth factor receptor-2. The patient was administered two adjuvant chemotherapy (AC) cycles (AC regimen: Pirarubicin, $40 \mathrm{mg}$, day 1 and 2; and CTX, 1 g i.v., on day 1 , then once every 3 weeks). However, following chemotherapy, the bone pain was not relieved. On May 24th, 2011, a positron emission tomography-computed tomography (PET-CT) examination revealed multiple metabolic enlarged lymph nodes with high fluorodeoxyglucose uptake under the left collarbone, in the mediastinum and in the two hilus pulmonis, as well as multiple nodules in the two lungs and pleuras at both sides, a subcutaneous nodule on the right back and multiple high metastatic lesions of the bone (Figs. 1 and 2). Subsequently, the treatment was changed from chemotherapy to endocrine therapy (anastrozole and goserelin regimen: Anastrozole, $1 \mathrm{mg}$ orally, daily; goserelin, $3.6 \mathrm{mg}$ subcutaneously, once every 28 days). Endocrine therapy treatment is on-going since June 29th, 2011, and zoledronic acid (4 mg i.v., once every 28 days) is also administered every 28 days.
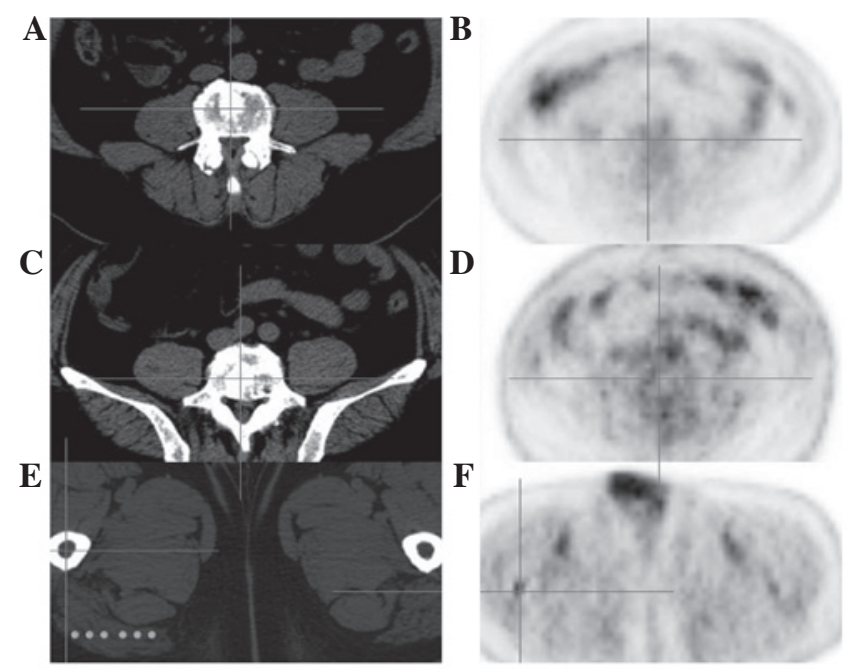

Figure 6. PET (right)-CT (left) scans (March 10th, 2014) revealed that the metabolism of osteoblastic metastases was back to normal, with greatly reduced levels compared with those prior to endocrine therapy. No evident changes were identified on axial $\mathrm{CT}$, however, corresponding axial PET images revealed almost normal ${ }^{18} \mathrm{~F}$-fluorodeoxyglucose uptake in the (A and $\mathrm{B}$ ) lumbar and (C and D) sacral vertebrae and (E and F) right femoral shaft. CT, computed tomography; PET, positron emission tomography.

The lower-limb bone pain was relieved following hormone therapy. On May 24th 2012, a PET-CT scan revealed that the disease had improved significantly. Fig. 3 demonstrates that the enlarged mediastinal lymph nodes were reduced in size, and the metabolic level was reduced. Fig. 4 indicates that the metabolic levels of the osteoblastic metastases were reduced also. The enlarged lymph nodes were much smaller, and the metabolic levels of the lymph nodes and osteoblastic metastases were reduced compared with previous examinations. The most commonly used markers for breast cancer are cancer antigen 153 and carcinoembryonic antigen, thus, levels of these antigens are presented in Table I. The increased levels of serum alkaline phosphatase are associated with bone metastasis. At the latest follow-up in 2014, the patient remained stable with no evidence of progression (Figs. 5 and 6).

The current study was approved by the ethics committee of China-Japan Union Hospital Affiliated to Jilin University, and written informed consent was obtained from the patient prior to the study.

\section{Discussion}

The presence of ectopic breast tissue is reported in 2-6\% of the general population with the majority of cases being located in 
the axillary region (3). Accessory breast carcinoma in males is extremely rare, with the most common clinical manifestation being accessory breast carcinoma of the axilla $(12,15)$. The most frequent histological type of this lesion is invasive ductal carcinoma (worldwide incidence, $72 \%)(16,17)$.

In the majority of studies, treatment regimens for accessory breast carcinoma follow the guidelines for breast cancer treatment $(9,10,11)$. Similar to breast cancer, accessory breast cancer is also surgically treated and supplemented with preoperative or postoperative chemotherapy (18). However, external radiotherapy should be considered in order to increase local control. Hormonal therapy is offered depending on the tumor and patients characteristics, following the same guidelines with anatomic breast carcinoma (19). Yamamura et al (8) have reported a case of male breast cancer originating in an accessory mammary gland in the axilla, which was successfully treated with hormone therapy (tamoxifen at $20 \mathrm{mg} /$ day) (8). In the case reported in the present study, the disease was not relieved following two cycles of adjuvant chemotherapy with the AC regimen. Based on the results of immunohistochemical analysis, which revealed that the tumor was ER $(+++)$ and PR (+++), the patient was treated with endocrine therapy (anastrozole and goserelin), which was an effective and tolerated therapy for the current patient.

Prognosis of accessory breast carcinoma is difficult to establish, primarily due to absent or limited follow-up data, and small sample sizes of previous studies (12). The disease follows similar prognostic indices as those of anatomic breast carcinoma (20). Certain authors have reported that carcinoma of accessory breast tissue may metastasize to the lymph nodes earlier and more frequently compared with anatomic breast carcinoma, since it usually occurs under the axilla or in the inguinal region, where there is an abundance of lymph nodes and capillaries $(19,21)$.

In conclusion, the present study reported a case of male accessory breast cancer in a patient with delayed diagnosis at a locally advanced stage; however, the patient was successfully treated with endocrine therapy. Currently, the patient is under follow-up observation, without any progression of the accessory breast cancer. The current case demonstrates that, although accessory breast cancer in males is extremely rare, the possibility of this disease should be considered when establishing a diagnosis. Investigation of further cases of accessory breast cancer in male patients will provide an improved understanding of the underlying mechanism, etiology, treatment and prognosis of this disease.

\section{References}

1. Fentiman IS, Fourquet A and Hortobagyi GN: Male breast cancer. Lancet 367: 595-604, 2006.

2. Gennari R, Curigliano G, Jereczek-Fossa BA, Zurrida S, Renne G, Intra M, Galimberti V, Luini A, Orecchia R, Viale G, et al: Male breast cancer: A special therapeutic problem. Anything new? (Review). Int J Oncol 24: 663-670, 2004.
3. Gutermuth J, Audring H, Voit C and Haas N: Primary carcinoma of ectopic axillary breast tissue. J Eur Acad Dermatol Venereol 20: 217-221, 2006.

4. Grossl NA: Supernumerary breast tissue: Historical perspectives and clinical features. South Med J 93: 29-32, 2000.

5. Matsuoka H, Ueo H, Kuwano H, Sugimachi K and Inokuchi K: A case of carcinoma originating from accessory breast tissue of the axilla. Gan No Rinsho 30: 387-391, 1984.

6. Takeyama H, Takahashi H, Tabei I, Fukuchi O, Nogi H, Kinoshita S, Uchida K and Morikawa T: Malignant neoplasm in the axilla of a male: Suspected primary carcinoma of an accessory mammary gland. Breast Cancer 17: 151-154, 2010.

7. Gao YG, Zhang SH and Wang Y: A case of accessory mammary cancer in a male patient and a literature review. Eur J Gynaecol Oncol 35: 452-455, 2014.

8. Yamamura J, Masuda N, Kodama Y, Yasojima H, Mizutani M, Kuriyama K, Mano M, Nakamori S and Sekimoto M: Male breast cancer originating in an accessory mammary gland in the axilla: A case report. Case Rep Med 2012: 286210, 2012.

9. Madej B, Balak B, Winkler I and Burdan F: Cancer of the accessory breast - a case report. Adv Med Sci 54: 308-310, 2009.

10. Kahraman-Cetintas S, Turan-Ozdemir S, Topal U, Kurt M, Gokgoz S, Saraydaroglu O and Ozkan L: Carcinoma originating from aberrant breast tissue. A case report and review of the literature. Tumori 94: 440-443, 2008.

11. Markopoulos C, Kouskos E, Kontzoglou K, Gogas G, Kyriakou V and Gogas J: Breast cancer in ectopic breast tissue. Eur J Gynaecol Oncol 22: 157-159, 2001.

12. Evans DM and Guyton DP: Carcinoma of the axillary breast. J Surg Oncol 59: 190-195, 1995.

13. Singletary SE and Connolly JL: Breast cancer staging: Working with the sixth edition of the AJCC Cancer Staging Manual. CA Cancer J Clin 56: 37-47, quiz 50-31, 2006.

14. Rusch VW, Asamura H, Watanabe $\mathrm{H}$, et al; Members of IASLC Staging Committee: The IASLC lung cancer staging project: A proposal for a new international lymph node map in the forthcoming seventh edition of the TNM classification for lung cancer. J Thorac Oncol 4: 568-577, 2009.

15. Amsler E, Sigal-Zafrani B, Marinho E and Aractingi S: Ectopic breast cancer of the axilla. Ann Dermatol Venereol 129: 1389-1391, 2002 (In French).

16. Visconti G, Eltahir Y, Van Ginkel RJ, Bart J and Werker PM: Approach and management of primary ectopic breast carcinoma in the axilla: Where are we? A comprehensive historical literature review. J Plast Reconstr Aesthet Surg 64: e1-e11, 2011.

17. Yerra L, Karnad AB and Votaw ML: Primary breast cancer in aberrant breast tissue in the axilla. South Med J 90: 661-662, 1997.

18. Hao JY, Yang CC, Liu FF, Yang YL, Li S, Li WD, Li YQ, Lang RG, Fan Y, Paulos E, et al: Accessory breast cancer occurring concurrently with bilateral primary invasive breast carcinomas: A report of two cases and literature review. Cancer Biol Med 9: 197-201, 2012.

19. Routiot T, Marchal C, Verhaeghe JL, Depardieu C, Netter E, Weber B and Carolus JM: Breast carcinoma located in ectopic breast tissue: A case report and review of the literature. Oncol Rep 5: 413-417, 1998.

20. Marshall MB, Moynihan JJ, Frost A and Evans SR: Ectopic breast cancer: Case report and literature review. Surg Oncol 3: 295-304, 1994.

21. Nihon-Yanagi Y, Ueda T, Kameda N and Okazumi S: A case of ectopic breast cancer with a literature review. Surg Oncol 20: 35-42, 2011. 